\title{
Model Experimental Study of Damage Effects of Ship Structures under the Contact Jet Loads of Bubble in a Water Tank
}

\author{
Hailong Chen, Baoyu Ni $\mathbb{D}$, Wenjin Hu $\mathbb{D}$, and Yanzhuo Xue \\ College of Shipbuilding Engineering, Harbin Engineering University, Harbin 150001, China \\ Correspondence should be addressed to Baoyu Ni; nibaoyu@hrbeu.edu.cn
}

Received 28 July 2017; Revised 27 November 2017; Accepted 20 December 2017; Published 23 January 2018

Academic Editor: Sergio De Rosa

Copyright (C) 2018 Hailong Chen et al. This is an open access article distributed under the Creative Commons Attribution License, which permits unrestricted use, distribution, and reproduction in any medium, provided the original work is properly cited.

\begin{abstract}
The damage effects of ship structures under the contact jet loads of bubble are studied by using an electric spark bubble as well as high-speed photography. A series of model experiments of ship structures under contact explosion was carried out in a water tank. On the one hand, we monitored the displacement and period of the oscillation of a hull plate of a ship model with a large bending rigidity. On the other hand, we observed the overall motion of a box-beam model with a small bending rigidity. The results show that when the distance parameter is less than 0.6 , the bubble jet will impact on the surface of the structure directly, which is defined as "contact bubble jet" herein. The contact bubble jet causes significant local loads on the ship and induces the "sagging moment" effect. This mainly results from the relatively negative bending moment caused by the bubble attached to the hull. With the increase of detonation distance, this negative bending moment decreases. As a result, the oscillation amplitude of the ship structure decreases sharply and the oscillation period reduces gradually.
\end{abstract}

\section{Introduction}

Underwater explosion (UNDEX) has been an effective way to attack surface vessels and underwater submarines. It is generally thought that UNDEX generates two types of loads mainly (Cole, 1948 [1]): the primary shock wave and the following bubble. A large number of studies have been done on the damage effects of shock waves in UNDEX, but a relatively lower number of studies have been done on those of bubbles. However, in recent years, more and more researchers (Kan et al., 2005 [2]; Riley, 2010 [3, 4]; Li et al., 2015 [5]; Cui et al., 2016 [6]; Zhang et al., 2017 [7]) have found that bubble loads could contribute a lot to the damage of the structures under some circumstances. For example, following the shock wave, the bubble expands and collapses many times while it migrates to the free surface, causing a whipping response of the structure (ISSC, 2015 [8]). Another feature of bubble behavior near rigid surfaces is the attraction to the wall during its contraction, which is also known as Bjerknes force (Bjerknes, 1966 [9]). Under this attraction force, the bubble moves towards the structure surface and distorts into a toroidal shape around the time of the bubble minima. This generates a high-speed water flow penetrating through the center of the toroid, which is generally referred to as a "bubble jet," and causes heavy damage on the structure. According to the load features and structural responses, the bubble loads can also be broadly divided into bubble fluctuation loads and bubble jet loads (Geers and Hunter, 2002 [10]) and so forth. In particular, when the bubble is very close to the structure, the bubble jet is one of the main reasons to cause the severe damage on the structure. Thus, we focus on the damage effects of the bubble jet mainly herein. For more information, the reader is referred to the Report of Committee V.5: Naval Vessel Design of ISSC (2015) [8].

In terms of damage effects caused by bubble loads, Kornfeld and Suvorov (1944) [11] should be the first researchers who mentioned that the collapse of a bubble would generate a high-speed "hydraulic blow," namely, a jet, which causes damage on the structures nearby. From then on, more and more researchers started to study the bubble jet and its damage effects by using experiments and numerical simulations. In experiments, Lauterborn and Bolle (1975) [12] studied the cavitation bubbles produced by a Q-switched ruby laser in the neighborhood of a solid boundary and photographed its motion and deformation by using a rotating-mirror camera. Time histories of distance and velocity of different points 
on the bubble surface were obtained and a jet configuration could be observed clearly as a result. Philipp and Lauterborn (1998) [13] studied the damage effect of a single laserproduced bubble on the aluminum specimen. Their focus was the effect of initial detonation distance on the erosion degree. Through a series of experiments, they found that the largest erosive force was caused by the collapse of a bubble in direct contact with the boundary and the pressure exerted on the material surface could be up to several GPa. Klaseboer et al. (2005) [14] studied the dynamics of an UNDEX bubble and its associated fluid-structure interaction. The experiments were carried out in a specially designed test pond and various rigid and resilient plates in circular shape were located near the bubble. The deformation of the plate was measured with a noncontact laser telemetry device. Bubble migration and jetting were discussed based on the experimental data. Brett and Yiannakopolous (2008) [15] studied the interaction of an UNDEX bubble and a nearby steel cylinder. The deformation and surface pressure of the cylinder were recorded and contrasted at different explosion distances. It was found that the damage of the cylinder caused by the collapse of the bubble was more severe than that of shock wave, generating a peak velocity almost twice that caused by the shock wave and causing significant plastic deformation. Gong et al. (2010) [16] analyzed the similarity relations of bubbles generated from different external sources, including electrical spark, laser, and UNDEX. Based on the improved Rayleigh equation, a scaling law between different types of bubbles was formulated, which provided a basis for the experimental observations of the spark, laser, and UNDEX generated bubbles. Lee et al. (2011) [17] investigated the damage of rigidly clamped square steel plates subjected to close-proximity UNDEX. High-explosive charges from $1.1 \mathrm{~g}$ to $50 \mathrm{~g}$ were used at different standoff distances to obtain different shock strengths and bubble collapse intensities. The experiment showed that bubble loads could not be ignored, although the main impulsive loads on the plate were due to the shock impact. When the standoff distance was less than twice the maximum free-field bubble radius, a strong interaction between the bubble and the target plate caused a rapid water jet impinging on the plate, resulting in additional loading and damage.

In mathematical modelling and numerical simulations, there is a relatively large body of literature on simulating the bubble near a rigid/elastic wall. It has been a common practice to assume that the flow is irrotational and thus can be described in terms of a velocity potential. By using potential flow theory, Brujan et al. (2002) [18] introduced a vortex sheet model to solve the doubly connected domain of the toroidal bubble caused by the penetration of the bubble jet. Wang et al. (2005) [19] introduced a vortex ring model at the toroidal bubble stage. Compared with the vortex sheet which needs to be traced carefully, vortex ring model has an advantage that one does not need to trace the vortex ring but only to make sure it always located in the toroidal bubble. Riley (2010) [4] studied the loading due to the bubble collapses by using a series of close-proximity UNDEX configurations, ranging from 0.2 up to 2.0 times the maximum free-field bubble radius. Cui et al. (2012) [20] adopted the equivalent method to transform the bubble jet into a truncated circular water column and used the finite-element software to simulate the deformation of a cabin under the bubble jet. Numerical simulation was compared with the experimental data and fair agreement was achieved. Jayaprakash et al. (2012) [21] adopted three-dimensional boundary-element method (BEM) to simulate the behaviors of a dynamic bubble near a vertical wall and study its jet characteristics. The fluid loading on the wall under the bubble jet was calculated, along with the detonation distances ranging from 0.25 up to 2.0 times the maximum free-field bubble radius. Wang et al. (2013) [22] used the delayed potential method to solve the impact response of cylindrical shells under bubble jets. It found the damage range of the bubble impact at various detonation positions relative to the cylindrical shell. Based on BEM, Ni et al. (2015) [23] simulated a high-speed bubble jet impacting on the rigid surface directly. A special treatment was introduced for dealing with the intersection point of solid, liquid, and gas and the fluid pressure and bubble deformation were simulated for the axisymmetric case during the direct impact. The variation law was investigated for the jet velocity versus standoff distance.

It seems from the previous studies that few researchers studied the impact of bubble jet loads very close to the structure in experiments, or contact impact on the structure, especially on the ship hull. Nevertheless, this phenomenon happens in the contact UNDEX, exerts high pressure on the ship, and causes severe damage. Thus, it is of significance to study the damage effect of the contact jet loads of the bubble. This forms the primary motivation for the present work and provides its key focus. Firstly, the definition of contact bubble jet is given. Then, some basic theories of model tests are followed. On this basis, two models of ship structures are adopted in experiments in a water tank and their structural responses under the contact bubble jet are studied.

\section{Definition of the "Contact Bubble Jet"}

Before defining the contact bubble jet, we define a dimensionless parameter-the distance parameter $\lambda$, which is the ratio between the standoff distance $h$ from the bubble center to the structure surface and the maximum free-field bubble radius $R_{m}$. As described in the Introduction, the deformation of the bubble and the velocity and the width of the jet to the wall may change significantly with the distance parameter. To study the effect of the distance parameter, we keep the initial pressure and buoyancy of the bubble constant while changing the initial detonation distance only and study the impact loads of the bubble jet to the rigid surface at different distances. According to the study of Ni et al. (2015) [23], the velocity of the bubble jet changes significantly with $\lambda$, as shown in Figure 1, where $\lambda$ varies from 0.15 to 1.6.

It can be seen from Figure 1 that there are two distinct trends for both numerical results (solid line) and experimental data (circle): when distance parameter $\lambda>0.6$, the jet velocity increases with $\lambda$; when $\lambda \leq 0.6$, the jet velocity decreases with $\lambda$. The trend in the range of $\lambda>0.6$ is consistent with those found in previous studies such as Blake and Gibson (1987) [24] and Wang (1998) [25]. The main 


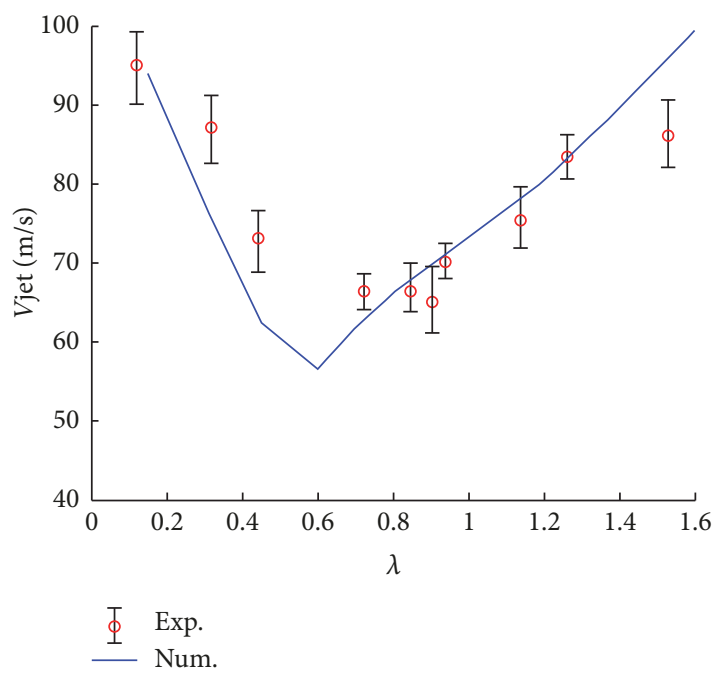

FIGURE 1: Variation of the jet velocity of the bubble versus distance parameter (Ni et al., 2015) [23].

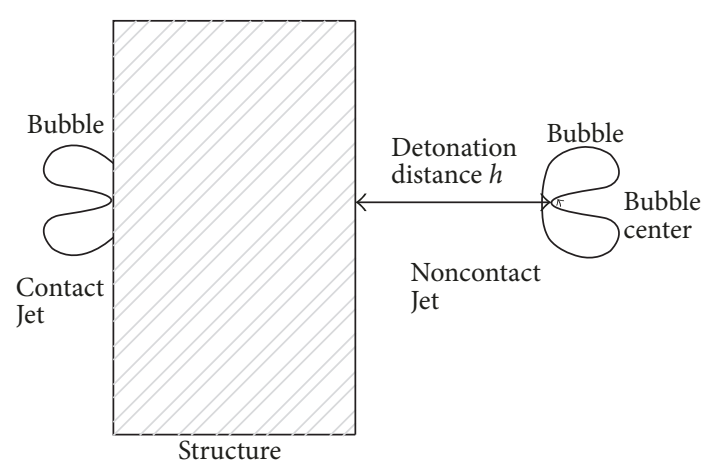

Figure 2: Sketch of "contact jet"' and "noncontact jet."

reason for the trend in this range is that the wall blocks the full development of the bubble and depresses the jet velocity as a result. Nevertheless, the jet velocity cannot increase infinitely as $\lambda$ increases. When $\lambda$ is large enough (around $\lambda>3$ ), the influence of the wall can be neglected and the behaviors of the bubble are similar to those of a bubble in an unbounded domain.

On the other hand, when $\lambda \leq 0.6$, the bubble is very close to the wall and the attraction force of the wall becomes much stronger, so the jet velocity gets larger as $\lambda$ decreases. In this range, the jet penetrates through one bubble side and impacts on the wall directly, rather than the other side of bubble. Therefore, based on the variation law shown in Figure 1, we define the bubble jet occurring within $\lambda \leq 0.6$ as "contact jet" while we define that beyond $\lambda>0.6$ as "noncontact jet" herein. The sketch of contact jet and noncontact jet is plotted in Figure 2. Although the velocity of contact jet may be less than that of noncontact jet, the contact jet is more destructive from the aspects of wall pressure, because the jet impacts on the wall directly, rather than on a water layer with a cushion effect. Thus, it is very important to study the damage effects of the contact jet.

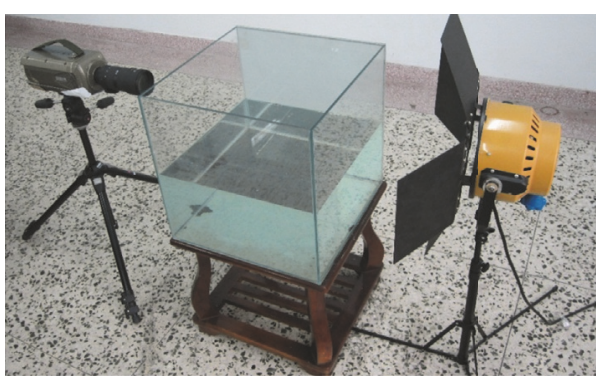

FIgURE 3: Experimental setup.

\section{Principles of Experiment}

In this paper, the bubble was produced by the electrical spark and its behavior was recorded by a high-speed camera. The high-speed camera is Phantom v12.1, which has the highest throughput of any complementary metal-oxidesemiconductor (CMOS) based digital high-speed camera. It can take more than 6,933 frames-per-second (fps) at full $1280 \times 720$ resolution and up to $1,000,000 \mathrm{fps}$ at reduced resolution. The minimum exposure is 290 nanoseconds. It has a built-in software, which can be used in the postprocessing. A series of experiments were performed in a water tank with dimensions $500 \mathrm{~mm} \times 500 \mathrm{~mm} \times 500 \mathrm{~mm}$. The depth of the de-aired water was $300 \mathrm{~mm}$. The electric poles were very fine copper wires. A direct current electric voltage $U=220 \mathrm{~V}$ and a capacitance $C=6600 \mu \mathrm{F}$ were considered for these experiments. The high-speed camera was set to $20,000 \mathrm{fps}$. A $2 \mathrm{~kW}$ light source was placed at the other end of the water tank to enhance the brightness of the view. The experimental setup (Zhang et al. [26-28]) is shown in Figure 3.

Two types of experimental models are adopted. One is a scaled model of a ship structure and the other is a beam-like structure, whose pictures will be provided in Figures 5 and 8 . The ship model is made of stainless steel and its main dimensions are $386.4 \mathrm{~mm}$ in length overall, $52.9 \mathrm{~mm}$ in extreme breadth, and $14.4 \mathrm{~mm}$ in draught. The geometric scale ratio is 295 . Here, we need to clarify that this model is not fully scaled from the prototype, although the outline is scaled. Due to the large-scale ratio 295, the thickness of hull steel of the model should be in the order of $0.1 \mathrm{~mm}$, which presents a huge challenge in construction. After overall consideration, we chose the stainless steel thick in the order of $1 \mathrm{~mm}$ to produce this ship model. As a result, the structural mechanical properties of the model, including inherent frequency and bending rigidity, do not satisfy the similarity relation. On the other hand, the beamlike structure is made of aluminum and its main dimensions are $200 \mathrm{~mm}$ in length overall, $39.4 \mathrm{~mm}$ in extreme breadth, and $4.8 \mathrm{~mm}$ in draft. The idea of this beam-like structure comes from a barge operating in Heilongjiang river system. The geometric scale ratio is 330 . However, we simplified its structure and adopted a simple box beam without frames. Key parameters of these two models are shown in Table 1.

The inherent frequency of the model is significant, which further depends on its bending rigidity. Strictly speaking, 
TABLE 1: Key parameters of the models.

\begin{tabular}{lcc}
\hline Main parameters & $\begin{array}{c}\text { Model 1: ship } \\
\text { structure }\end{array}$ & $\begin{array}{c}\text { Model 2: beam-like } \\
\text { structure }\end{array}$ \\
\hline Length overall $L(\mathrm{~mm})$ & 386.4 & 200 \\
Extreme breadth $B(\mathrm{~mm})$ & 52.9 & 39.4 \\
Draught $D(\mathrm{~mm})$ & 14.4 & 4.8 \\
Geometric scale ratio & 295 & 330 \\
Material & Stainless steel & Aluminum \\
\hline
\end{tabular}

the bending rigidity and inherent frequency of the models should be measured by using special apparatus. However, our laboratory is lacking these special apparatuses at present and this paper focuses on the contact jet loads of the bubble, so we give a method to estimate the bending rigidity and inherent frequency of the models roughly. Some assumptions need to be given in advance. Firstly, we assume that the model can be considered as a beam with uniform distributed mass and constant bending rigidity. Secondly, the model is simplified as a single-degree-of-freedom system with a single inherent frequency. On this basis, the model with length $l$ is hinged support at both ends and a weight with mass $M$ is exerted at the midpoint of the model. Based on the knowledge of mechanics of materials (Beer et al., 2012) [29], one can obtain the maximum deflection $\delta$ of the beam at the midpoint as

$$
\delta=\frac{g l^{3}}{48 \mathrm{EI}}\left(M+\frac{5}{8} m\right)
$$

where $g$ is the acceleration due to gravity, EI is the bending rigidity of the model, also known as $K$, and $m$ is the mass of the model. By measuring $\delta$, one can obtain the bending rigidity easily through

$$
K=\mathrm{EI}=\frac{g l^{3}}{48 \delta}\left(M+\frac{5}{8} m\right) .
$$

In addition, if we assume the static deflection curve as the deflection curve of the model in vibration, we can obtain the inherent frequency of the model $\omega_{n}$ (Timoshenko, 2008) [30] as

$$
\omega_{n}=\sqrt{\frac{48 \mathrm{EI}}{(M+(5 / 8) m) l^{3}}} .
$$

Alternatively, if we assume that the deflection curve of the model in vibration has a sinusoidal form $y(x)=$ $A \sin ((\pi / l) x)$, we can obtain the inherent frequency of the model $\omega_{n}$ (Timoshenko, 2008) [30] as

$$
\omega_{n}=\sqrt{\frac{48 \mathrm{EI}}{(M+(1 / 2) m) l^{3}}} .
$$

By using (2) and (3) or (4), one can estimate the bending rigidity and inherent frequency of the models roughly. Here, we need to declare that this method is quite rough, because the vibration of the structure must be elastic and there

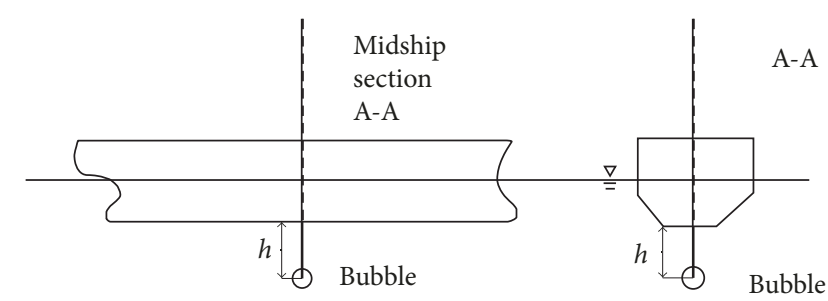

FIGURE 4: Sketch of the bubble generation position relative to the model.

should be different order bending mode frequencies. Besides, when the structure floats on the water, the added mass of water should be considered and the wet frequencies need to be solved. Thus, we just use (2)-(4) qualitatively rather than quantitatively. Furthermore, we define a frequency ratio between the inherent frequency $\omega_{n}$ of the model and the frequency $\omega_{b}$ of the bubble loads as

$$
\eta=\frac{\omega_{n}}{\omega_{b}}
$$

which will be used in Section 4 .

As mentioned above, the built-in software of the camera Phantom v12.1 is used in the postprocessing. By using this built-in software, the dimensionless displacement of any point in the experimental view can be measured directly. A reference of the length should be given to obtain the corresponding dimensional displacement. A ruler is usually placed next to the model and shot by the camera before each experiment, which would be used as a reference of the length for the experimental data. The accuracy of the experimental data is related to two main aspects. One is minimum scale of the reference of the length, which was $0.01 \mathrm{~mm}$ in our experiments. The other is uncertainty assessment in the experiment. Based on the study of Zhang et al. (2015) [26] by using the same experimental setup and analysis methods, it showed that the uncertainty in such experiments was about $0.3 \% \sim 0.6 \%$, which can be accepted and applies to this paper also.

The spark bubble is generated at a distance $h$ right below the model, along the intersection line of midship section and central longitudinal section of the model, as shown in Figure 4. The specific distance will be provided and discussed in Section 4. There is another challenge during the experiment. The initial internal pressure of the bubble is difficult to measure directly in the experiment. To obtain the initial internal pressure of the spark bubble, an experiment of a single bubble in the free field is needed before its interaction with the structure. The initial radius $R_{0}$ and maximum radius $R_{m}$ of the bubble in the free field can be measured directly. The motion equation of the spherical bubble is Rayleigh-Plesset equation (Rayleigh, 1917) [31]:

$$
\ddot{R} R+\frac{3}{2} \dot{R}^{2}=\frac{1}{\rho}\left(P_{c}+P_{0}\left(\frac{R_{0}}{R}\right)^{3 \tau}-\frac{2 \sigma}{R}-P_{\infty}\right),
$$

where $R$ is the bubble radius, $\dot{R}$ and $\ddot{R}$ are the first- and second-order derivatives of bubble radius with time, respectively, $\rho$ is density of water, $P_{c}$ is the saturated vapor pressure 


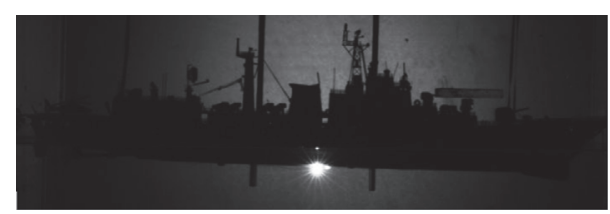

(a) $t=0.125 \mathrm{~ms}$

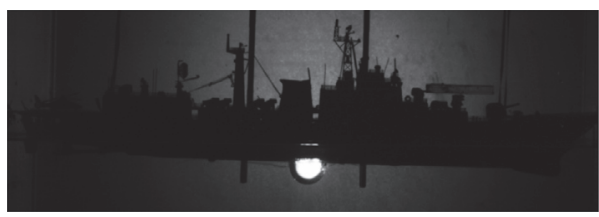

(c) $t=2.125 \mathrm{~ms}$

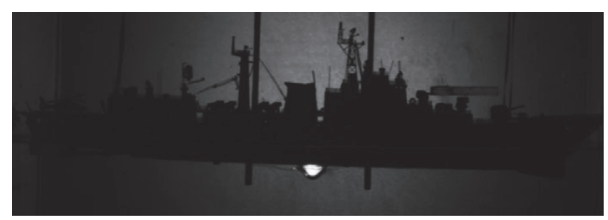

(e) $t=4.125 \mathrm{~ms}$

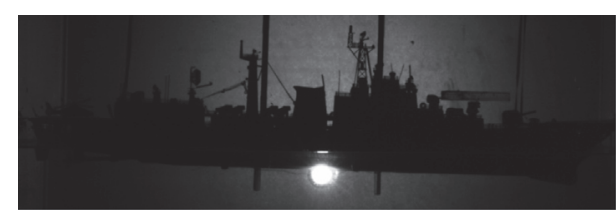

(b) $t=1.125 \mathrm{~ms}$

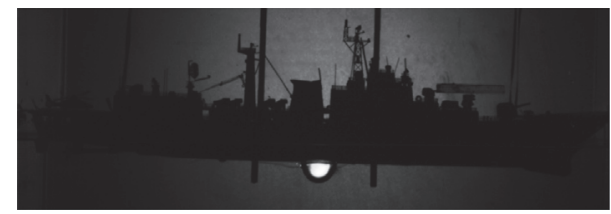

(d) $t=3.250 \mathrm{~ms}$

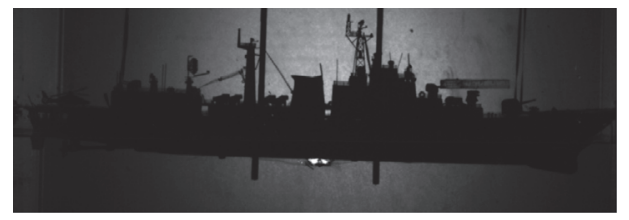

(f) $t=4.625 \mathrm{~ms}$

FIGURE 5: Dynamic response of a scaled ship model under contact bubble jet $(\lambda \approx 0.1)$.

of condensable gases, $P_{0}$ is the initial internal pressure of the bubble, $\tau$ is the specific heat ratio of noncondensable gas, $\sigma$ is the surface-tension coefficient of fluid, and $P_{\infty}$ is the reference pressure at infinity. Without special instructions, herein $\rho$ is taken as $1000 \mathrm{~kg} / \mathrm{m}^{3}, P_{c}$ is taken as $2338 \mathrm{~Pa}, \tau$ is taken as $1.25, \sigma$ is taken as $7.3 \times 10^{-2} \mathrm{~N} / \mathrm{m}$, and $P_{\infty}$ is taken as the standard atmospheric pressure.

Rewriting (6) in the form of an integral, one has

$$
\frac{\rho}{2}\left[\frac{d}{d R}\left(R^{3} \dot{R}^{2}\right)\right]=P_{0}\left(R_{0}\right)^{3 \tau} R^{2-3 \tau}-\Delta P R^{2}-2 \sigma R,
$$

where $\Delta P=P_{\infty}-P_{c}$.

Integrating (7) from $R_{0}$ to a certain radius $R$, one has

$$
\begin{aligned}
\frac{\rho}{2}\left(\dot{R}^{2}-\dot{R}_{0}^{2}\right)= & \frac{P_{0}}{3-3 \tau}\left(\frac{R_{0}^{3 \tau}}{R^{3 \tau}}-\frac{R_{0}^{3}}{R^{3}}\right)+\frac{\Delta P}{3}\left(\frac{R_{0}^{3}}{R^{3}}-1\right) \\
& +\sigma\left(\frac{R_{0}^{2}}{R^{3}}-\frac{1}{R}\right) .
\end{aligned}
$$

Considering $\dot{R}_{0}=0$ when the bubble is initially generated ( $R=R_{0}$ ) and $\dot{R}_{m}=0$ when the bubble achieves its maximum in the free field ( $\left.R=R_{m}\right)$ and substituting $R=R_{m}$ into (8), one has

$$
\frac{\varsigma}{3-3 \tau}\left(\bar{R}_{0}^{3 \tau}-\bar{R}_{0}^{3}\right)+\frac{1}{3}\left(\bar{R}_{0}^{3}-1\right)+\frac{1}{\mathrm{We}}\left(\bar{R}_{0}^{2}-1\right)=0,
$$

where $\varsigma=P_{0} / \Delta P$ is the strength parameter, We $=R_{m} \Delta P / \sigma$ is the Weber number, and $\bar{R}_{0}=R_{0} / R_{m}$ is the dimensionless initial radius, which can be determined by the measured initial radius $R_{0}$ and maximum radius $R_{m}$. By solving (9), one can obtain the expression of the strength parameter:

$$
\varsigma=\left[\bar{R}_{0}^{3}-1+\frac{3}{\mathrm{We}}\left(\bar{R}_{0}^{2}-1\right)\right] \frac{\tau-1}{\bar{R}_{0}^{3 \tau}-\bar{R}_{0}^{3}} .
$$

Equation (10) can be used to solve the strength parameter of the electric spark bubble. Here is one exception: when $\bar{R}_{0}=1$, the denominator on the right-hand side of the equation is 0 , so (10) cannot be used to solve the strength parameter directly then. However, $\bar{R}_{0}=1$ denotes that the initial radius of the bubble is the maximum radius, so the bubble is in a static equilibrium state and $\varsigma=1+2 /$ We can be obtained according to the force balance condition. For UNDEX bubbles, Cole (1948) [1] summed up the experimental data and provided empirical formulas for the initial internal pressure and the maximum radius as functions of the charge and the depth of explosion. For more details, please refer to Cole (1948) [1].

The bubble is generated by an electric spark in this paper, so it is a qualitative but not quantitative test for real UNDEX attacking ships, where the bubble is generated by underwater explosive products. However, previous studies (Gong et al., 2010) [16] show that there exists a similarity relation between the spark bubble and the UNDEX bubble. Herein, we do not derive this similarity relation and the specific forms could be found by referring to Wang et al. (2013) [32].

\section{Discussions of the Experimental Results}

By using the experimental devices described above, two typical structures are addressed in this section: a scaled model of a ship structure and a box-beam-like structure. The dynamic responses of the structures under contact bubble jet are analyzed.

4.1. Experiment of a Ship Model Structure. Figure 5 shows the results of the ship model under contact bubble jet, where dimensional time is marked under each figure. The spark bubble's initial radius $R_{0} \approx 1.77 \mathrm{~mm}$, the equivalent maximum radius $R_{m} \approx 11.7 \mathrm{~mm}$, and the initial explosion 


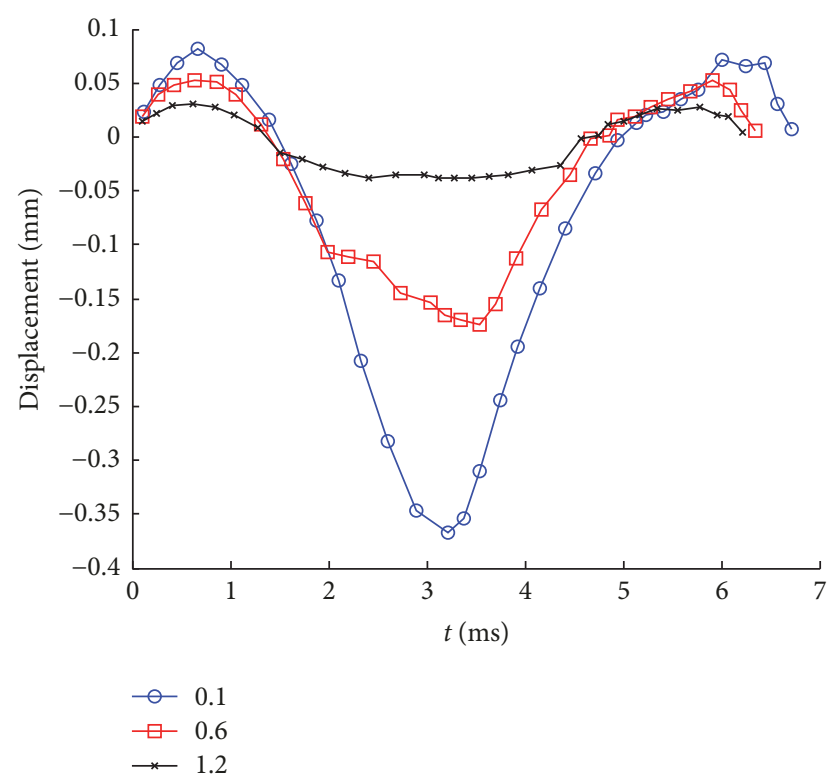

FIGURE 6: Time history of displacement of the center point on the hull plate.

distance $h \approx 1.22 \mathrm{~mm}$. It can be obtained that distance parameter $\lambda=h / R_{m} \approx 0.1$ and strength parameter $\varsigma \approx 106.3$ based on (10).

As shown in Figure 5(a), when $=0.125 \mathrm{~ms}$, the bubble just is generated and expands rapidly under its high pressure inside. When $t=1.125 \mathrm{~ms}$ in Figure 5(b), the bubble continues to expand and the hull plate moves upward under the action of the bubble loads. At the moment of $t=2.125 \mathrm{~ms}$ in Figure 5(c), the bubble reaches its maximum volume with a hemispherical shape and attaches to the hull plate closely. Except the local deformation in the bubble coverage region, the overall deformation of the ship is slight. When $t=$ $3.250 \mathrm{~ms}$ in Figure 5(d), the bubble begins to contract in a hemispherical shape. In Figure 5(e), the bubble shrinks further and the hull presents a sagging motion under the negative bending moment of the bubble. It can be seen clearly in Figure 5(f) at $t=4.625 \mathrm{~ms}$ that the contact bubble jet impacts on the hull plate directly. Afterwards, a toroidal bubble (Zhang et al., 2015) [33] would be generated; however, investigating that deformation is beyond the scope of this paper.

Based on the method described in Section 3, we can estimate the overall stiffness of the ship model is large and its inherent frequency $\omega_{n s}$ is high, which is much greater than the low frequency $\omega_{b}$ of the bubble loads. The frequency ratio $\eta_{s}=\omega_{n s} / \omega_{b} \gg 1$ for this ship model, so the overall deformation of this ship model is slight. Nevertheless, for contact explosions, the main structural response is the local oscillation of the plate in the bubble coverage region. Therefore, the displacement of the center point on the hull plate is provided in Figure 6, as a function of time. Three curves are given corresponding to three distance parameters $\lambda$ of $0.1,0.6$, and 1.2 , respectively. For the condition $\lambda=0.1$, the outer plate of the hull shows a slight upward motion

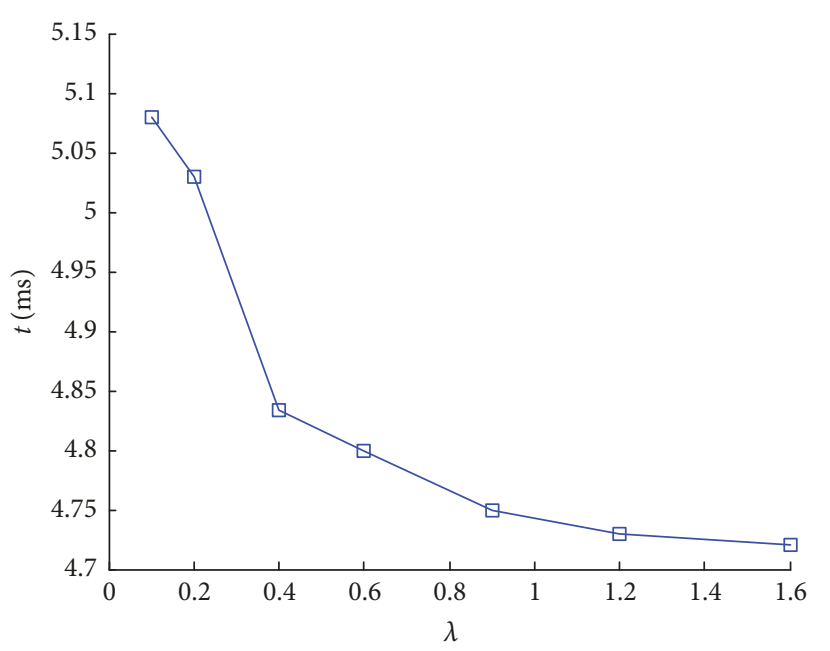

FIGURE 7: Variation of the oscillation period of the hull plate versus the distance parameter.

under the action of the shock wave generated by the electric spark initially. In the bubble contraction stage, the outer plate of hull presents a substantial downward movement under the action of negative bending moment caused by the relatively negative pressure of the bubble. The amplitudes of the upward and downward movement are asymmetrical. After the impact of contact bubble jet, the hull plate moves up gradually and reverts to the upward motion again, before reaching a peak value. In addition, as shown in Figure 6, the displacement amplitude of the hull plate decreases along with the increase of the distance parameter. The amplitudes of the upward and downward movement get closer as the distance parameter rises. According to Li et al. (2001) [34], the hull will be in a whipping motion under the noncontact explosion loads. For the ship model with large bending rigidity, when the bubble is away from the structure, or $\lambda$ is large, the bubble fluctuation loads and noncontact bubble jet dominate and cause a whipping response of the ship. Under such circumstance, the safety of the longitudinal strength of the ship was threatened by the overall whipping response. In contrast, when the bubble is near the structure, or $\lambda$ is small, the contact bubble jet loads dominate and induce severe local oscillation of the hull plate right above the bubble. Large displacement of the hull plate threatens the safety of the local strength of the ship.

The oscillation period of the hull plate is defined as the time interval between two peaks in the displacement curve. Figure 7 shows the variation trend of the oscillation period of the hull plate along with the distance parameter. The oscillation period of the hull decreases gradually with the increase of detonation distance. The main reason for this phenomenon is that the period of bubble pulsation decreases gradually with an increase of the distance parameter (Ni et al., 2015) [23]. The oscillation frequency of the structure is positively related to that of the external loads in the forced oscillation, so the oscillation period of the hull decreases gradually as the bubble pulsates quicker under a larger distance parameter. 


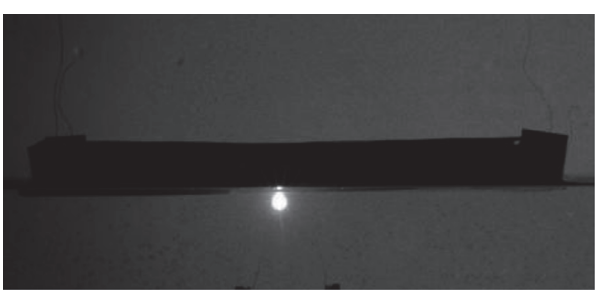

(a) $t=0.213 \mathrm{~ms}$

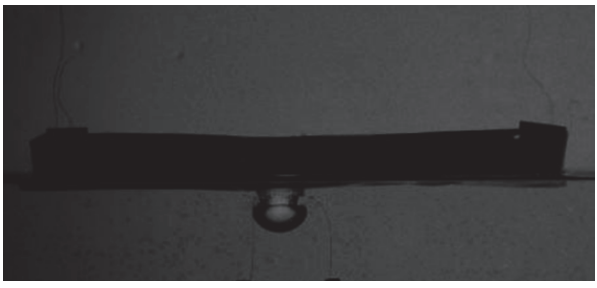

(c) $t=2.988 \mathrm{~ms}$

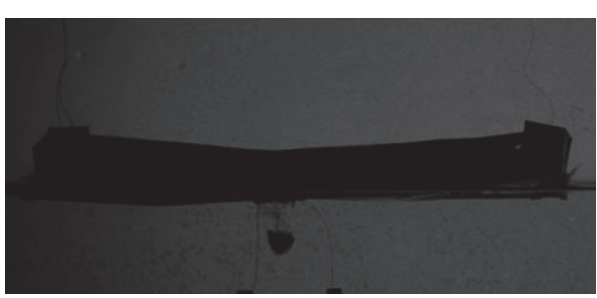

(e) $t=4.589 \mathrm{~ms}$

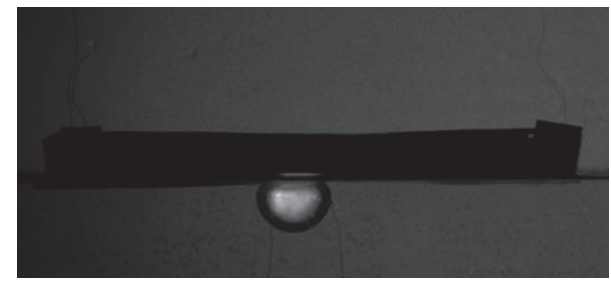

(b) $t=1.814 \mathrm{~ms}$

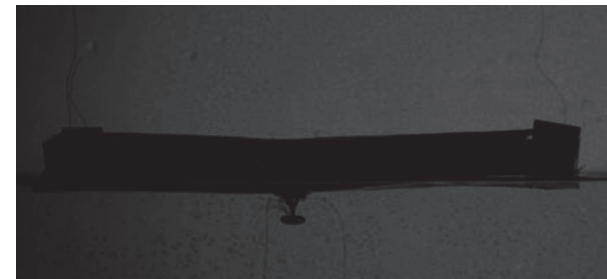

(d) $t=3.522 \mathrm{~ms}$

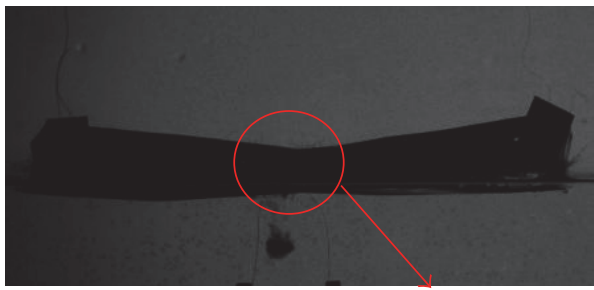

Plastic hinge

(f) $t=5.656 \mathrm{~ms}$

FIGURE 8: Dynamic response of a box-beam-like structure under contact bubble jet $(\lambda \approx 0.1)$.

4.2. Experiment of a Box-Beam-Like Structure. Considering that the bending rigidity of the ship model is large and the overall deformation response is slight, we study a box-beamlike structure with small bending rigidity EI (or $K$ ) and low inherent frequency $\omega_{n b}$ in this section. The initial radius of the spark bubble $R_{0} \approx 1.8 \mathrm{~mm}$, the equivalent maximum radius $R_{m} \approx 12.0 \mathrm{~mm}$, and the initial explosion distance $h \approx 1.22 \mathrm{~mm}$. Thus, one has the distance parameter $\lambda \approx 0.1$ and the strength parameter $\varsigma \approx 97.3$ based on (10).

Figure 8 shows the dynamic response of a box-beamlike structure under contact explosion with $\lambda \approx 0.1$. In Figure $8(\mathrm{a})$, around $=0.213 \mathrm{~ms}$, the bubble is in its initial expansion stage and the box beam is pushed up under the action of the expansion bubble loads. When the bubble reaches the maximum volume at $t=1.814 \mathrm{~ms}$ in Figure 8(b), it presents a hemispherical shape and attaches to the hull. At this moment, the box beam reaches its maximum hogging state. Then, in Figure 8(c) at $=2.988 \mathrm{~ms}$, the bubble starts to contract and the box beam starts to change from hogging to sagging states under the action of the negative bending moment of the bubble. At $t=3.522 \mathrm{~ms}$ in Figure 8(d), the bubble collapses to the minimum, when the box beam reaches the maximum sagging state. Then, the bubble enters the second expansion stage. The middle part of the box beam begins to rise again around $t=4.589 \mathrm{~ms}$ as visible in Figure 8(e). It can be seen clearly from Figure 8(f) that a "plastic hinge" (Fatt et al., 1996 [35]) is generated at the middle

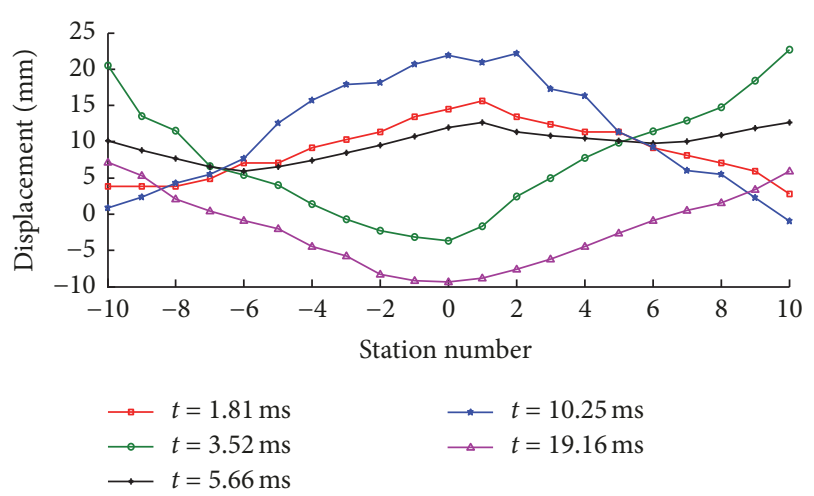

Figure 9: Whipping motion of the box beam under the contact explosion.

part of the box beam around $t=5.656 \mathrm{~ms}$ under the action of the bubble loads, which denotes that the box beam has entered the plastic failure stage.

Figure 9 shows the overall displacement curves of the baseplate of the box beam at different times, where the total length of the box beam has been divided into 20 stations. The expansion and collapse of the bubble arouse the hogging and sagging of the box beam with small bending rigidity. The box beam has large-amplitude displacements in the middle part and has a plastic deformation finally, as shown in Figure 8(f). 
Based on the study of Li et al. (2001) [34], the amplitude of the displacement of the ship under middle- and farfield explosion is small and the whipping motion is mainly based on the first-order vertical mode of vibration. Unlike what happens for middle- and far-field explosion, not only the overall ship movement but also local oscillation in the bubble coverage area can be found for near-field explosion. Furthermore, the contact bubble causes a negative bending moment acting on the hull for a relatively long time, which results in a severe sagging damage to the hull.

\section{Conclusions}

Through the experiments in a water tank, the damage effects of ship structures under the loading of contact bubble jet are studied. The oscillation displacement and period of the ship structure with large bending rigidity are monitored, and the overall motion of the box-beam-like structure with small bending rigidity is observed. The main conclusions are drawn as follows:

(1) The jet of the bubble very close to the wall impacts the wall surface directly, rather than on the water layer between the bubble and the wall. Therefore, two bubble jet modes are proposed herein: "noncontact jet" and "contact jet." Based on the experimental observations and numerical calculations, $\lambda \approx 0.6$ is determined as the critical value between these two modes, below which is the "contact jet" region.

(2) "Noncontact jet" mainly affects the total longitudinal strength of the ship, while "contact jet" has a different damage effect on the nearby structures. Because the contact jet impacts the surface of the structure directly, it usually causes the local oscillation of the hull plate and threatens the safety of the local structural strength of the ship. With the increase of explosion distance, the oscillation amplitude of the outer hull plate decreases sharply and the oscillation period decreases gradually.

(3) For the contact explosion, a local oscillation can be found in the coverage area of the bubble besides the overall displacement of the ship. Under the negative bending moment of the bubble loading for a relatively long time, the contact-explosion bubble causes a serious sagging motion of the hull and even generates a "plastic hinge" at the middle of the structure with a small bending rigidity.

The results of the present study can be generalized in a qualitative way, because the experimental models are not fully scaled. Although the bending rigidity and inherent frequency of the models are not similar to those of their prototypes, the responses of the structures subjected to a contact bubble jet are general. Thus, the conclusions of this analysis can be used to predict the behaviors of a ship subjected to a contact bubble jet qualitatively, which is the scope of this paper. In the next step, the fully scaled models with a similar bending rigidity and an inherent frequency will be constructed and adopted to improve the accuracy of the prediction. Then, the results of the scaled models will be used to predict the prototypes quantitatively under similarity principles.

\section{Conflicts of Interest}

The authors declare that there are no conflicts of interest regarding the publication of this paper.

\section{Acknowledgments}

This work is supported by the National Key Research and Development Program of China (2016YFE0202700), the National Natural Science Foundation of China (nos. 51639004, 51679044, 51579054, and 41606213), the Fundamental Research Funds for the Central Universities (no. HEUCFP201701), and the 111 Project in HEU, to which the authors are most grateful.

\section{References}

[1] R. H. Cole, Underwater Explosion, Princeton University Press, New Jersey, NJ, USA, 1948.

[2] K.-K. Kan, J. H. Stuhmiller, and P. C. Chan, "Simulation of the collapse of an underwater explosion bubble under a circular plate," Shock and Vibration, vol. 12, no. 3, pp. 217-225, 2005.

[3] M. Riley, "Analytical solution for predicting underwater explosion gas bubble behavior," Defence R \& D Canada-Atlantic 237, 2010.

[4] M. Riley, "Modeling gas bubble behaviour and loading on a rigid target due to close-proximity underwater explosions: comparison to tests conducted at DRDC suffield," Defence R \& D Canada-Atlantic 238, 2010.

[5] S. Li, Y. B. Li, and A. M. Zhang, "Numerical analysis of the bubble jet impact on a rigid wall," Applied Ocean Research, vol. 50, pp. 227-236, 2015.

[6] P. Cui, A. M. Zhang, and S. P. Wang, "Small-charge underwater explosion bubble experiments under various boundary conditions," Physics of Fluids, vol. 28, no. 11, Article ID 117103, 2016.

[7] A. M. Zhang, W. B. Wu, Y. L. Liu, and Q. X. Wang, "Nonlinear interaction between underwater explosion bubble and structure based on fully coupled model," Physics of Fluids, vol. 29, no. 8, p. 082111, 2017.

[8] C. Guedes Soares and Y. Garbatov, "Report of Committee V.5: Naval vessel design," in Proceedings of the 19th international ship and offshore structures congress, 2015.

[9] Bjerknes, Fields of Force, Columbia University Press, Columbia, 1966.

[10] T. L. Geers and K. S. Hunter, "An integrated wave-effects model for an underwater explosion bubble," The Journal of the Acoustical Society of America, vol. 111, no. 4, pp. 1584-1601, 2002.

[11] M. Kornfeld and L. Suvorov, "On the destructive action of cavitation," Journal of Applied Physics, vol. 15, no. 6, pp. 495-506, 1944.

[12] W. Lauterborn and H. Bolle, "Experimental investigations of cavitation-bubble collapse in the neighbourhood of a solid boundary," Journal of Fluid Mechanics, vol. 72, no. 2, pp. 391399, 1975.

[13] A. Philipp and W. Lauterborn, "Cavitation erosion by single laser-produced bubbles," Journal of Fluid Mechanics, vol. 361, pp. 75-116, 1998. 
[14] E. Klaseboer, K. C. Hung, C. Wang et al., "Experimental and numerical investigation of the dynamics of an underwater explosion bubble near a resilient/rigid structure," Journal of Fluid Mechanics, vol. 537, pp. 387-413, 2005.

[15] J. M. Brett and G. Yiannakopolous, "A study of explosive effects in close proximity to a submerged cylinder," International Journal of Impact Engineering, vol. 35, no. 4, pp. 206-225, 2008.

[16] S. W. Gong, S. W. Ohl, E. Klaseboer, and B. C. Khoo, "Scaling law for bubbles induced by different external sources: theoretical and experimental study," Physical Review E, vol. 82, Article ID 056317, pp. 1-11, 2010.

[17] J. J. Lee, M. J. Smith, J. Huang, and G. T. Paulgaard, "Deformation and rupture of thin steel plates due to cumulative loading from underwater shock and bubble collapse," Shock and Vibration, vol. 18, no. 3, pp. 459-470, 2011.

[18] E. A. Brujan, G. S. Keen, A. Vogel, and J. R. Blake, "The final stage of the collapse of a cavitation bubble close to a rigid boundary," Physics of Fluids, vol. 14, no. 1, pp. 85-92, 2002.

[19] Q. X. Wang, K. S. Yeo, B. C. Khoo, and K. Y. Lam, "Vortex ring modelling of toroidal bubbles," Theoretical and Computational Fluid Dynamics, vol. 19, no. 5, pp. 303-317, 2005.

[20] J. Cui, S. M. Li, C. Huang, X. L. Yao, and A. M. Zhang, "Application research of jet-impact model in an underwater explosion experiment," Chinese Journal of High Pressure Physics, vol. 26, no. 5, pp. 523-530, 2012.

[21] A. Jayaprakash, C.-T. Hsiao, and G. Chahine, "Numerical and experimental study of the interaction of a spark-generated bubble and a vertical wall," Journal of Fluids Engineering, vol. 134, no. 3, Article ID 031301, 2012.

[22] S.-P. Wang, J. Guo, Z.-Y. Zhang, H.-L. Chen, and F. Sun, "Dynamic response of a cylindrical shell under bubble jet loading," Explosion and Shock Waves, vol. 33, no. 4, pp. 337-343, 2013.

[23] B. Y. Ni, A. M. Zhang, and G. X. Wu, "Numerical and experimental study of bubble impact on a solid wall," Journal of Fluids Engineering, vol. 137, no. 3, Article ID 031206, 2015.

[24] J. R. Blake and D. C. Gibson, "Cavitation bubbles near boundaries," Annual Review of Fluid Mechanics, vol. 19, pp. 99-123, 1987.

[25] Q. X. Wang, "The evolution of a gas bubble near an inclined wall," Theoretical and Computational Fluid Dynamics, vol. 12, no. 1, pp. 29-51, 1998.

[26] A. M. Zhang, P. Cui, J. Cui, and Q. X. Wang, "Experimental study on bubble dynamics subject to buoyancy," Journal of Fluid Mechanics, vol. 776, pp. 137-160, 2015.

[27] S. Zhang, S. P. Wang, and A. M. Zhang, "Experimental study on the interaction between bubble and free surface using a highvoltage spark generator," Physics of Fluids, vol. 28, no. 3, Article ID 032109, 2016.

[28] S. Zhang, A. M. Zhang, S. P. Wang, and J. Cui, "Dynamic characteristics of large scale spark bubbles close to different boundaries," Physics of Fluids, vol. 29, no. 9, p. 092107, 2017.

[29] F. P. Beer, E. R. Johnston, J. T. DeWolf, and D. F. Mazurek, Mechanics of Materials, McGraw-Hill Education Press, New York, NY, USA, 6th edition, 2012.

[30] S. Timoshenko, Vibration Problems in Engineering, Wolfenden Press, Calif, CA, USA, 2008.

[31] J. W. Rayleigh, "On the pressure developed in a liquid during the collapse of a spherical cavity," Philosophical Magazine, vol. 34, no. 200, pp. 94-98, 1917.
[32] S. P. Wang, F. Zhu, C. Wu, and A. M. Zhang, "Similarity Method in Scaled Experiment of Interaction between Underwater Explosion Bubbles and Ships," Shipbuilding of China, pp. 30-38, 2013.

[33] A. M. Zhang, S. Li, and J. Cui, "Study on splitting of a toroidal bubble near a rigid boundary," Physics of Fluids, vol. 27, no. 6, Article ID 062102, 2015.

[34] Y. J. Li, X. C. Zhang, Y. S. Wu, and Q. F. Chen, "Whipping response of ship hull induced by underwater explosion bubble," Shipbuilding of China, vol. 42, no. 3, p. 1, 2001.

[35] M. S. H. Fatt, T. Wierzbicki, M. Moussouros, and J. Koenig, "Rigid-plastic approximations for predicting plastic deformation of cylindrical shells subject to dynamic loading," Shock and Vibration, vol. 3, no. 3, pp. 169-181, 1996. 


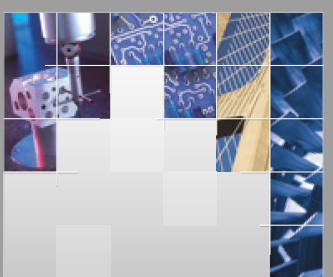

\section{Enfincering}
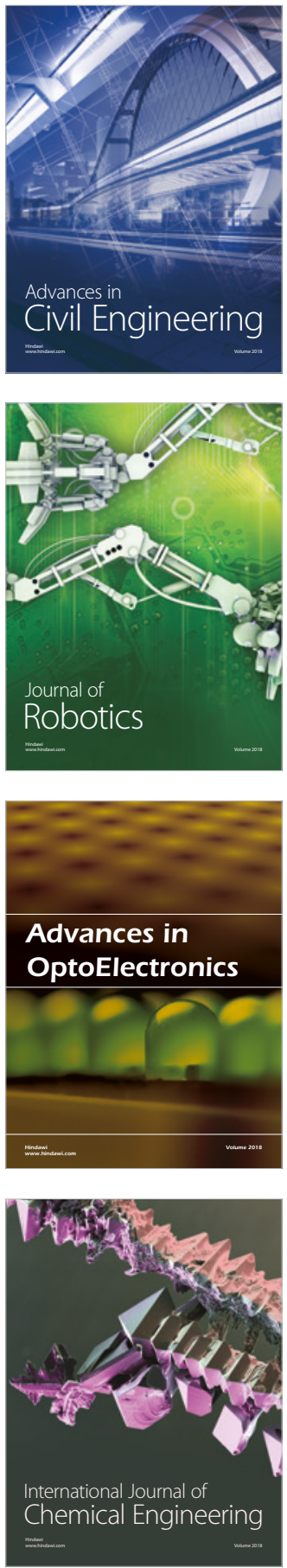

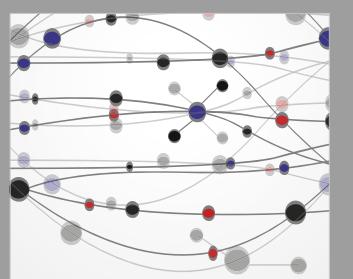

\section{Rotating \\ Machinery}

The Scientific World Journal

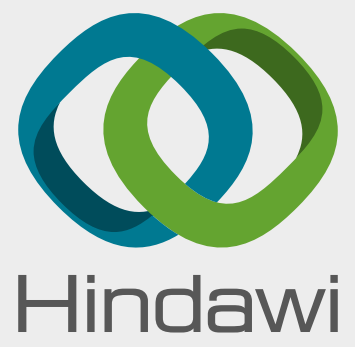

Submit your manuscripts at

www.hindawi.com
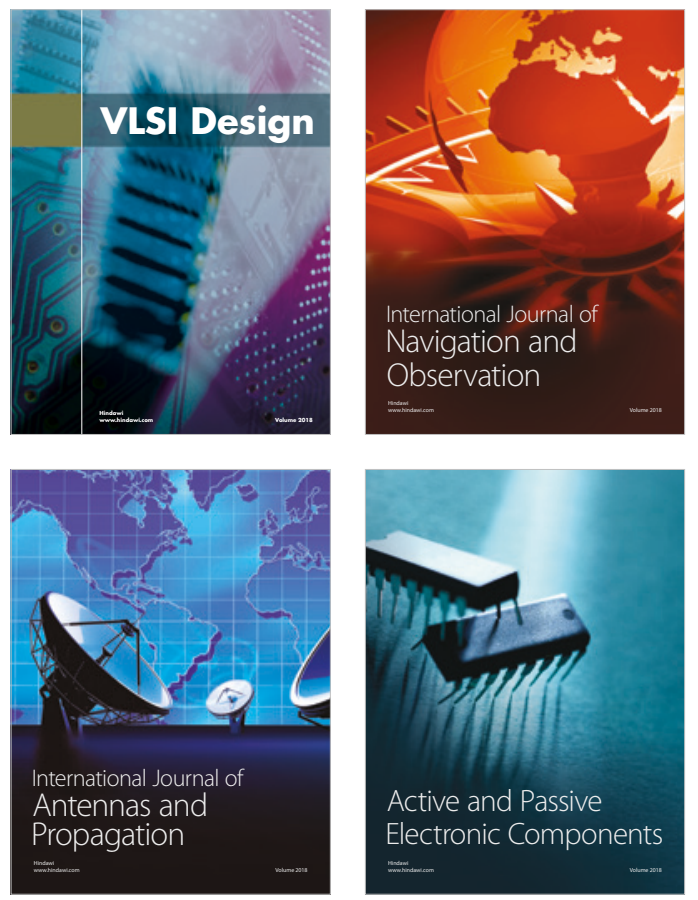
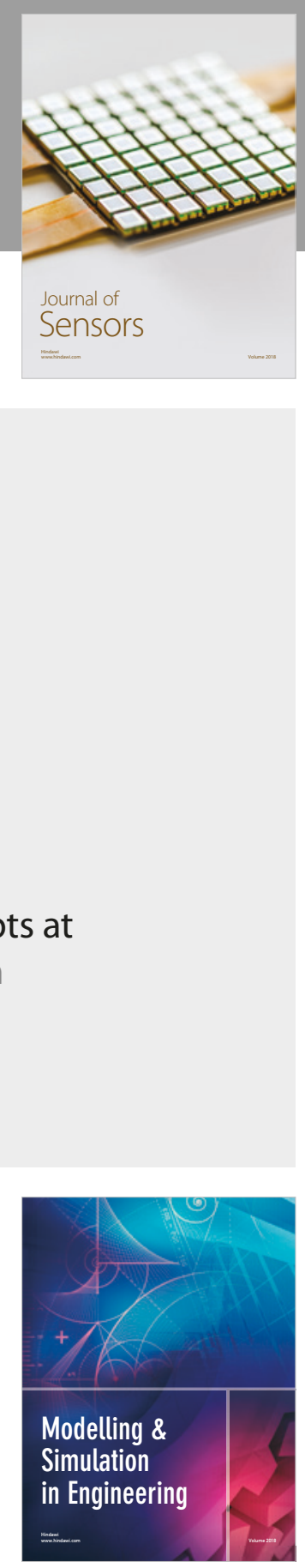

\section{Advances \\ Multimedia}
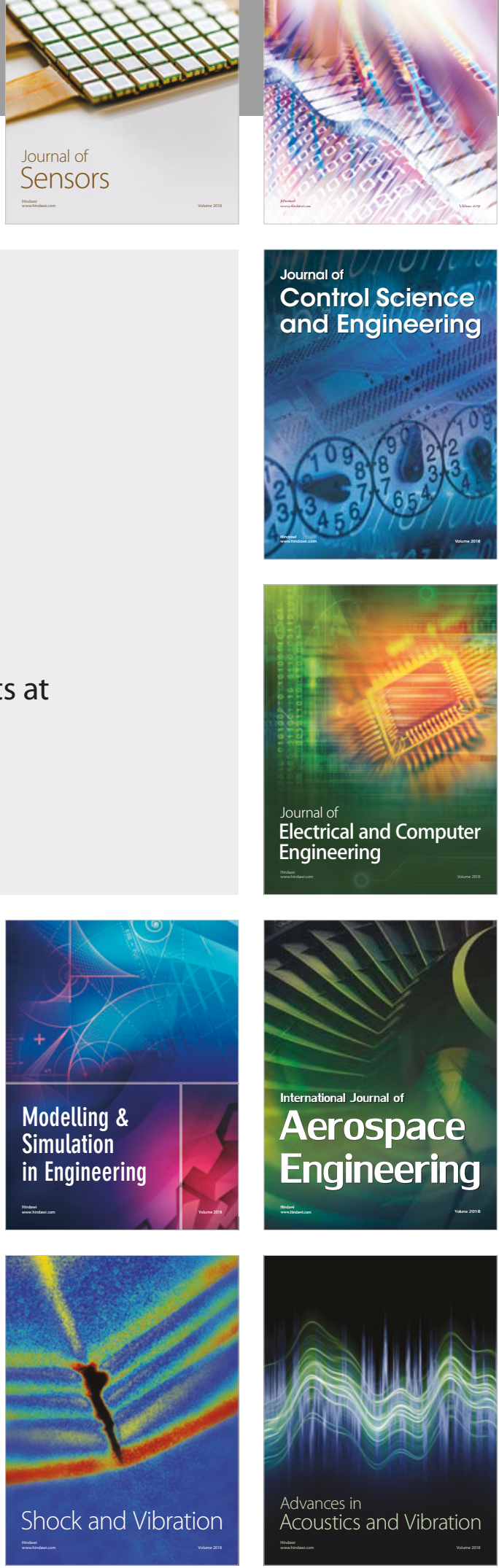